\title{
A phase II trial of concurrent chemoradiotherapy with weekly paclitaxel and carboplatin in advanced oesophageal carcinoma
}

\author{
$\mathrm{Yi} \mathrm{Xia}^{1,2} \cdot$ Yun-hai Li ${ }^{2} \cdot$ Yun Chen ${ }^{1} \cdot \mathrm{Qi} \mathrm{Liu}^{1} \cdot$ Jun-hua Zhang ${ }^{1} \cdot$ Jia-ying Deng ${ }^{1} \cdot$ Ta-shan Ai $^{1} \cdot$ Han-ting Zhu ${ }^{1}$. \\ Harun Badakhshi ${ }^{3} \cdot$ Kuai-Le Zhao ${ }^{1}$
}

Received: 16 August 2017 / Accepted: 2 January 2018 / Published online: 12 February 2018

(c) The Author(s) 2018. This article is an open access publication

\begin{abstract}
Background This study was performed to assess the efficacy and feasibility of definitive chemoradiotherapy consisting of weekly doses of combined paclitaxel and carboplatin concurrent with radiation therapy, followed by 2 cycles of consolidation chemotherapy for advanced esophageal carcinoma.

Methods Eligibility criteria included local, advanced, newly diagnosed and postoperative local regional lymph node metastasis; Eastern Cooperative Oncology Group (ECOG) score $\leq 2$; and adequate organ function. Patients received concurrent chemoradiation therapy consisting of radiotherapy (50.4 Gy/28 Fx or $61.2 \mathrm{~Gy} / 34 \mathrm{Fx}$ ) and concurrent paclitaxel (50 mg/ $\mathrm{m}^{2}$ ) and carboplatin (area under the curve, AUC $=2$ ) on days 1, 8, 15, 22 and 29. The two-cycle consolidation chemotherapy protocol was paclitaxel $\left(175 \mathrm{mg} / \mathrm{m}^{2}\right)$ plus carboplatin $(\mathrm{AUC}=5)$ administered on days 57 and 85 , after concurrent chemoradiotherapy.

Results Between August 2013 and February 2015, 65 patients with oesophageal carcinoma were enrolled in the study; 34 $(52.3 \%)$ were newly diagnosed and $31(47.6 \%)$ had postoperative local regional lymph node metastasis. The median overall survival time was 21.7 months (95\% confidence interval [CI] 16.7-26.6), and the median progression-free survival time was 12.1 months (95\% CI 9.0-15.3). A total of 96.9\% (63/65) and 67.6\% (44/65) patients completed $\geq 5$ cycles and all 7 cycles of chemotherapy, respectively. A total of $93.8 \%$ (61/65) patients completed radiation therapy. The 1- and 2-year overall survival rates were 73.7 and $42.0 \%$, respectively. The 1- and 2-year progression-free survival rates were 50.6 and $31.1 \%$, respectively. Grade 3-4 toxicity during chemoradiotherapy included neutropenia (24.5\%), thrombocytopenia (4.6\%), fatigue (1.5\%), anaemia (1.5\%), radiation dermatitis (1.5\%), pneumonitis (1.5\%), oesophagitis (4.6\%) and vomiting (1.5\%).

Conclusions In patients with locally advanced oesophageal cancer, the combination of weekly doses of paclitaxel and carboplatin was well tolerated and produced comparable results. A three-arm randomised phase III trial (NCT02459457) comparing paclitaxel in combination with cisplatin, carboplatin or 5-fluorouracil with concurrent radiotherapy is on-going at our hospital.
\end{abstract}

Keywords Oesophageal carcinoma $\cdot$ Paclitaxel $\cdot$ Carboplatin $\cdot$ Chemoradiotherapy

Yi Xia and Yun-hai Li share first authorship.

Kuai-Le Zhao

kuaile_z@sina.com

1 Department of Radiation Oncology, Fudan University, Shanghai Cancer Center, 270 Dong'An Road, Shanghai 200032, China

2 Department of Radiation Oncology, Fudan University, Shanghai Cancer Center Minhang Branch, 106RuiLi Road, Shanghai 200240, China

3 Department of Clinical Radiation Oncology, Ernst von Bergmann Medical Center, D-14467 Potsdam, Germany

\section{Introduction}

Oesophageal cancer is highly malignant with a propensity for high rates of local recurrence and metastasis. Although significant advances in staging and treatment of patients have recently been seen, this malignancy is still lethal for the majority of patients [1]. Definitive concurrent chemoradiotherapy is the standard treatment for non-surgery patients with oesophageal cancer, and the use of cisplatin with 5-fluorouracil (5-FU) is the most common chemotherapy regimen [2]. However, after definitive chemoradiation therapy, 5-year survival rates are poor (26\%) [3]. In addition, 
$20 \%$ of patients who received chemoradiation experienced life-threatening side effects, and chemotherapy could not be completed in $>40 \%$ of the patients [4]. Thus, more sensitive, less toxic chemotherapy regimens are urgently needed.

Paclitaxel is a promising agent in oesophageal cancer, with response rates of approximately $32 \%$ with single-drug treatment in locally advanced and metastatic patients [5]. Paclitaxel is a mitotic inhibitor that blocks cells in the G2M phase of the cell cycle, the most radiosensitive phase, with a sensitising enhancement ratio of 1.48 [6]. The investigation of a paclitaxel-based regimen and administration route is important for improving survival and reducing side effects. Effective and well-tolerated chemotherapy can prolong overall survival and improve quality of life.

The combination of paclitaxel and carboplatin (TC) with concurrent radiotherapy has been tested in patients with advanced non-small-cell lung cancer [7-9]. In several of these studies, the combination of paclitaxel and carboplatin was given weekly with concurrent radiotherapy, followed by two or four cycles of consolidation chemotherapy. The overall response rate varied from 71 and $79 \%$. The major toxicity was oesophagitis: in $10-46 \%$ of the patients a grade 3 or 4 oesophagitis was found. Treatment with paclitaxel and carboplatin and concurrent radiotherapy can be given on an outpatient basis, which is advantageous. Furthermore, this regimen is probably less toxic than cisplatin-based therapy. Van Meerten et al. reported a phase 2 study of neoadjuvant chemoradiotherapy (nCRT) consisting of weekly administration of carboplatin and paclitaxel with concurrent radiotherapy [10]. The regimen was associated with a low rate of serious toxic effects, and a complete resection with no tumor within $1 \mathrm{~mm}$ of the resection margins (R0) was achieved in all patients who underwent resection. In the CROSS study, which included 366 patients with esophageal and gastrooesophageal junctional tumors, the complete resection rate was increased from $69 \%$ with surgery alone to $92 \%$ with the combined therapy, with no increase in the preoperative mortality rate. Ultimately, the nCRT in this trial's population provided a highly significant $34 \%$ reduction of the risk of death, as well as a significant $42 \%$ reduction of the risk of relapse [11].

In comparison with cisplatin, carboplatin has a lower incidence of neurotoxicity, and is easier to administer in outpatients. Pharmacokinetic studies of paclitaxel administered with carboplatin demonstrate that area under the curve (AUC)-guided dosing can be accurately predicted by the Calvert formula. On the basis of these data, we began a phase II feasibility study to evaluate the overall survival (OS), progression-free survival (PFS) and toxicities in advanced oesophageal cancer patients treated with weekly paclitaxel and carboplatin and concurrent radiotherapy.

\section{Patients and methods}

\section{Patient selection}

The eligibility criteria for the study were as follows: (1) age $\leq 75$ years; (2) Eastern Cooperative Oncology Group (ECOG) score 0-2; (3) cytologically or histologically confirmed oesophageal cancer; and (4) locally advanced, newly diagnosed patients with T2-4NxM0-1a or TxNxM1b (supraclavicular lymph node metastases for middle and lower thoracic oesophageal cancer or mediastinal lymph node metastases for cervical oesophageal cancer; without organ metastases according to the 6th edition of UICC) or postoperative local regional lymph node metastasis patients who did not receive chemotherapy or radiotherapy.

Patients were required to meet the following laboratory criteria: adequate bone marrow function (neutrophil count $>2.0 \times 10^{9} / \mathrm{L}$, white blood cell count $>4.0 \times 10^{9} / \mathrm{L}$ and platelet count $>100 \times 10^{9} / \mathrm{L}$ ), normal renal function and normal liver function. Patients with tracheoesophageal fistula, organ metastasis or complete obstruction were not eligible for this study. All patients underwent complete examination and nutritional assessment. The disease evaluation included upper endoscopy, chest and abdominal computed tomography (CT), ultrasonography and barium oesophagram examination to eliminate distant organ metastasis.

\section{Ethical considerations}

Written informed consent was obtained from all patients before pre-study assessments, and the study protocol was approved by the Ethics Committee of the Cancer Hospital Affiliated to Fudan University.

\section{Treatment schedule}

The chemotherapy regimen consisted of a combination of paclitaxel and carboplatin. Concurrent chemotherapy was administered from the first day of radiotherapy and comprised five cycles of paclitaxel $\left(50 \mathrm{mg} / \mathrm{m}^{2}\right)$ in a 3-hour infusion and infused carboplatin $(\mathrm{AUC}=2)$ on days 1 , $8,15,22$ and 29 . Thirty minutes before treatment with paclitaxel, patients were pre-medicated with $10 \mathrm{mg}$ of dexamethasone and $300 \mathrm{mg}$ of cimetidine intravenously and $25 \mathrm{mg}$ of promethazine intramuscularly. The twocycle consolidation chemotherapy protocol was paclitaxel $\left(175 \mathrm{mg} / \mathrm{m}^{2}\right)$ plus carboplatin $(\mathrm{AUC}=2)$ on weeks 9 and 13. Pre-treatment before paclitaxel treatment was as follows: $25-\mathrm{mg}$ promethazine intramuscular injection at $0.5 \mathrm{~h}, 27$ tablets of dexamethasone administered orally $(0.75 \mathrm{mg} /$ tablet $)$ at 12 and $6 \mathrm{~h}$ before treatment, and $300 \mathrm{mg}$ of cimetidine administered intravenously at 


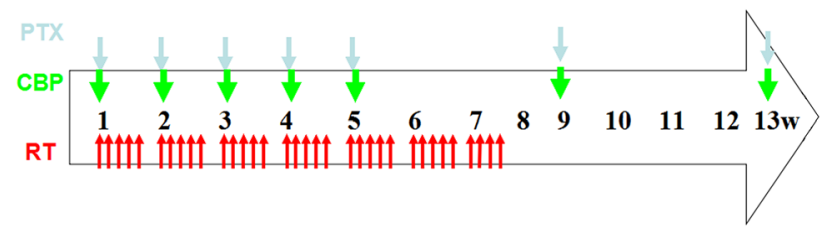

Fig. 1 Treatment schedule. Radiation therapy (RT): total dose of $61.2 \mathrm{~Gy} / 34 \mathrm{Fx}, 1.8 \mathrm{~Gy} / \mathrm{Fx}, 5$ days/week. Concurrent chemotherapy: paclitaxel (PTX) $50 \mathrm{mg} / \mathrm{m}^{2}$ and carboplatin (CBP) AUC $=2$ on days 1, 8, 15, 22 and 29. Consolidation chemotherapy: PTX $175 \mathrm{mg} / \mathrm{m}^{2}$ plus carboplatin $\mathrm{AUC}=5$ was given on week 9 and 13

$0.5 \mathrm{~h}$ before paclitaxel treatment. If grade $\geq 3$ haematological toxicity occurred and persisted, chemotherapy was suspended until recovery, and the regimen dose was sequentially reduced by $25 \%$. The treatment modality is illustrated in Fig. 1.

Radiation therapy was administered using intensitymodulated radiation therapy (IMRT). Planning was performed using a Megavoltage simulator with a photon energy of $6 \mathrm{MV}$. For previously untreated diagnosed patients and recurrent patients, the gross tumour volume (GTV) was defined as the volume of the primary tumour observed on oesophageal barium exam, upper digestive endoscopy and CT. Metastatic lymph nodes were defined as lymph nodes $\geq 1 \mathrm{~cm}$ in the shortest axis and $\geq 5 \mathrm{~mm}$ in the tracheo-oesophageal groove. The clinical target volume (CTV) was defined by adding 3-cm margins of proximally and distally uninvolved oesophagus without including lateral margins and lymph nodes. The planning target volume (PTV) was calculated by adding $1-\mathrm{cm}$ margins around the CTV, but the margins were reduced when the PTV covered the spinal cord. All patients were treated with a total dose of either $61.2 \mathrm{~Gy}$ in 34 fractions or $50.4 \mathrm{~Gy}$ in 28 fractions. The lower dose was used when patients had abdominal lymph node metastasis or tumour in excess of the limitations of normal organs, such as the lungs, spiral cord and intestines.

Plan optimisation was as follows: (1) $99 \%$ of the PTV was covered by $95 \%$ of the prescribed dose; (2) $95 \%$ of the PTV volume was covered by the prescribed dose; (3) the maximum dose did not exceed $110 \%$ of the prescribed dose in a continuous volume of $<1 \mathrm{~cm}^{3}$ in the PTV; and (4) the maximum dose of the PTV did not exceed $110 \%$ of the prescribed dose in a continuous volume of $<1 \mathrm{~cm}^{3}$. The normal tissue constraints of the critical organs were as follows: a maximum spinal cord point dose of $\leq 45 \mathrm{~Gy}$; lung V20 (percentage of total lung volume receiving $\geq 20 \mathrm{~Gy}$ ) of $\leq 30 \%$ and mean lung of $\leq 16 \mathrm{~Gy}$; mean heart dose of $\leq 30 \mathrm{~Gy}$; and maximum intestine dose of $\leq 50 \mathrm{~Gy}$.

\section{Follow-up}

In the first and second year after treatment completion, the patients were seen at the outpatient clinic every 3 months for a history and clinical examination. During the third year after treatment, follow-up occurred every 6 months until year 5. If applicable, disease recurrence, late toxic effects or death was documented. Adverse reactions to chemoradiotherapy were evaluated according to the National Cancer Institute Common Toxicity Criteria version 4.0 (NCI-CTC 4.0).

\section{Statistical methods}

The primary end point of this study was OS. Secondary end points comprised assessment of safety and compliance, PFS and local control (LC). Local failure was defined as recurrence or persistence within the radiation therapy PTV. Recurrence outside the treatment volume was defined as distant. OS was calculated from the first day of chemoradiation therapy to the time of the last follow-up or death. PFS was defined as the time from chemoradiotherapy day 1 to progression, death or last follow-up. We hypothesised that the addition of paclitaxel and carboplatin would increase the 1-year overall survival rate. A sample size of 59 was required to detect an increase in the 1-year survival rate from 57 to $73 \%$ with a power of $80 \%$ and $\alpha$ error (two-sided test) of 0.05 . A $10 \%$ adjustment for drop-outs resulted in a sample size of 65 patients. Data were analysed according to the intention-to-treat population. Kaplan-Meier curves were fitted to estimate the OS and failure-free local control rates. Median survival estimates were calculated. Statistical analyses were performed using SPSS 20.0.

\section{Results}

\section{Patient characteristics}

Between August 2013 and February 2015, 65 patients were enrolled in our study. Figure 2 shows the progression through the study phases. The baseline characteristics of the 65 patients are shown in Table 1. The median patient age was 61 years (range $47-74$ ). Nine patients (11.3\%) had other malignant tumours, and 59 patients $(90.7 \%)$ were men. ECOG performance status was $0-1$ in 59 patients and 2 in 6 patients. The most common tumour histology was squamous (90.7\%). Of all patients, 34 (52.3\%) were newly diagnosed, and 31 (47.6\%) had postoperative regional lymph node metastasis and had not received prior chemotherapy and radiotherapy. Among all newly diagnosed patients, 2/34 (5.8\%) patients were stage II, 10/34 (29.4\%) were stage III and 22/34 (64.7\%) were stage IV. Two had tumours in the cervical region, 4 in the upper thoracic region, 16 in the 
Fig. 2 CONSORT diagram of patient progress through the study

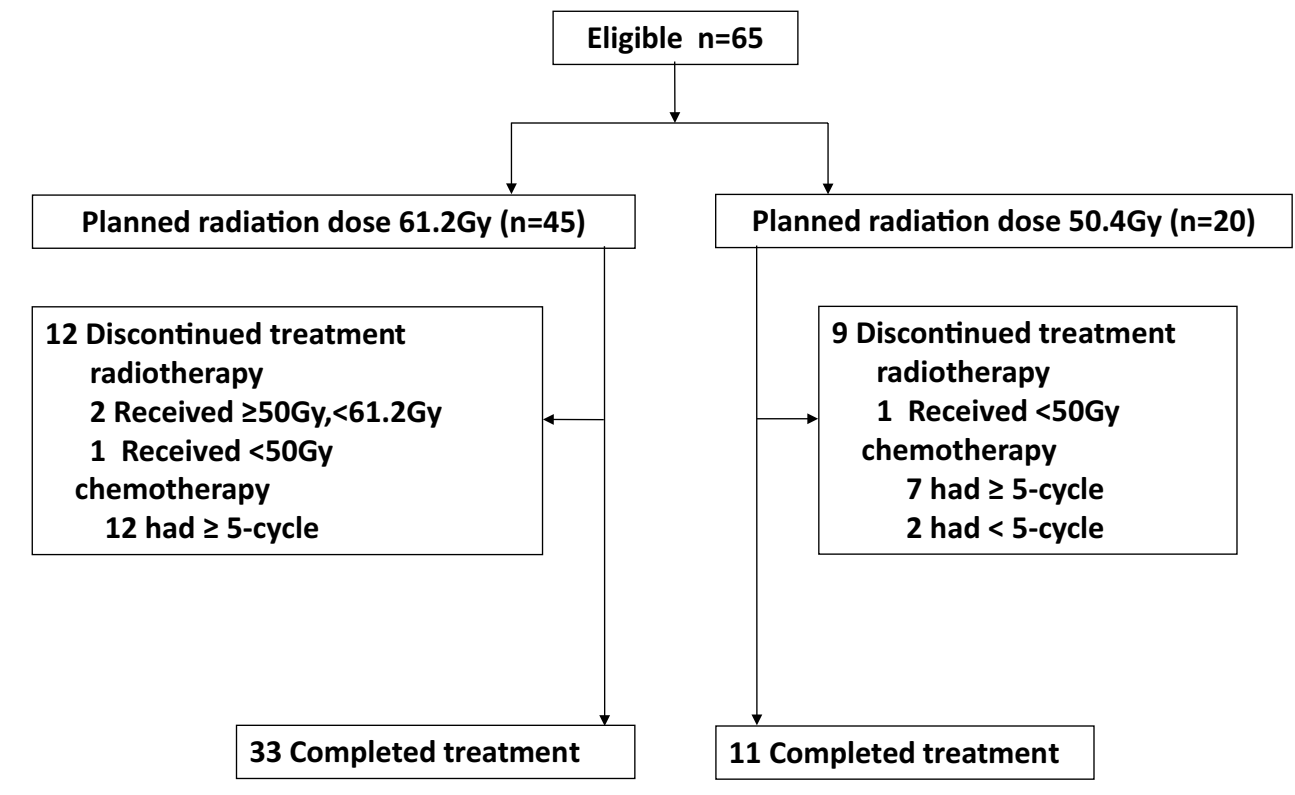

Table 1 Clinical characteristics of 65 patients analysed in this study

\begin{tabular}{|c|c|}
\hline Clinical parameter & $n(\%)$ \\
\hline \multicolumn{2}{|l|}{ Age (years) } \\
\hline Mean & 61 \\
\hline Range & $47-74$ \\
\hline \multicolumn{2}{|l|}{ Gender } \\
\hline Male & $59(90.7)$ \\
\hline Female & $6(9.2)$ \\
\hline \multicolumn{2}{|l|}{ Histology } \\
\hline Squamous & $59(90.7)$ \\
\hline Adenocarcinoma & $4(6.1)$ \\
\hline Poorly differentiated carcinoma & $2(3.0)$ \\
\hline Location of newly diagnosed tumor & $36(52.3)$ \\
\hline Cervical & $2(3.0)$ \\
\hline Upper thoracic & $4(6.1)$ \\
\hline Middle thoracic & $16(24.6)$ \\
\hline Lower thoracic & $10(15.3)$ \\
\hline Synchronous multiple primary & $2(3.0)$ \\
\hline \multicolumn{2}{|l|}{$\mathrm{ECOG}^{\mathrm{a}}$ score } \\
\hline $0-1$ & $59(90.7)$ \\
\hline 2 & $6(9.2)$ \\
\hline \multicolumn{2}{|l|}{ Stage $\left(\right.$ UICC $^{\mathrm{b}} 6$ th edition $)$} \\
\hline Newly diagnosed & $34(52.3)$ \\
\hline II & $2(3.0)$ \\
\hline III & $10(15.3)$ \\
\hline IV & $22(33.8)$ \\
\hline Postoperative $\mathrm{LNM}^{\mathrm{c}}$ & $31(47.6)$ \\
\hline \multicolumn{2}{|l|}{ Planned dose of radiation } \\
\hline $61.2 \mathrm{~Gy}$ & $45(69.2)$ \\
\hline 50.4 Gy & $20(30.7)$ \\
\hline
\end{tabular}

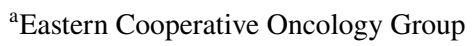

${ }^{\mathrm{b}}$ Union for International Cancer Control

${ }^{\mathrm{c}}$ Lymph node metastasis middle thoracic region, 10 in the lower thoracic region and 2 in multiple synchronous primary sites.

\section{Feasibility}

Among all patients, 45 had a planned radiation dose of 61.2 Gy and 42 (93.3\%) completed radiotherapy treatment. Of the 20 patients with a planned radiation dose of $50.4 \mathrm{~Gy}$, $19(95.0 \%)$ patients finished the complete radiotherapy regimen. A total of 61 patients $(93.8 \%)$ completed treatment based on our definition for completing the radiotherapy regimen. Four (6.1\%) patients did not finish radiotherapy because of heart disease (1), grade III oesophagitis (1), esophageal fistula (1) or esophageal bleeding (1).

$\mathrm{A} \geq 5$-cycle dose of concurrent chemotherapy with paclitaxel and carboplatin during radiotherapy was given to 63 patients (96.9\%), and a full dose of 7 cycles of chemotherapy was given to 44 patients (67.6\%). Two patients (3.0\%) did not finish the 5-cycle dose of concurrent chemotherapy because of grade III thrombocytopenia (1) or ventricular arrhythmia (1).

\section{OS and PFS rates}

The median follow-up time was 26.3 months. At the time of our analyses, 27 patients were alive and 19 patients had no evidence of disease progression. The 1- and 2-year OS rates were 73.7 and $42.0 \%$, respectively. The 1- and 2-year PFS rates were 50.6 and $31.1 \%$, respectively. The median OS time was 21.7 months (95\% confidence interval [CI] 16.7-26.6) and the median PFS time was 12.1 months (95\% CI 9.0-15.3). Kaplan-Meier curves for the OS and PFS time are shown in Fig. 3. The 1- and 2-year local control rates were 75.4 and $68.9 \%$, respectively. The 

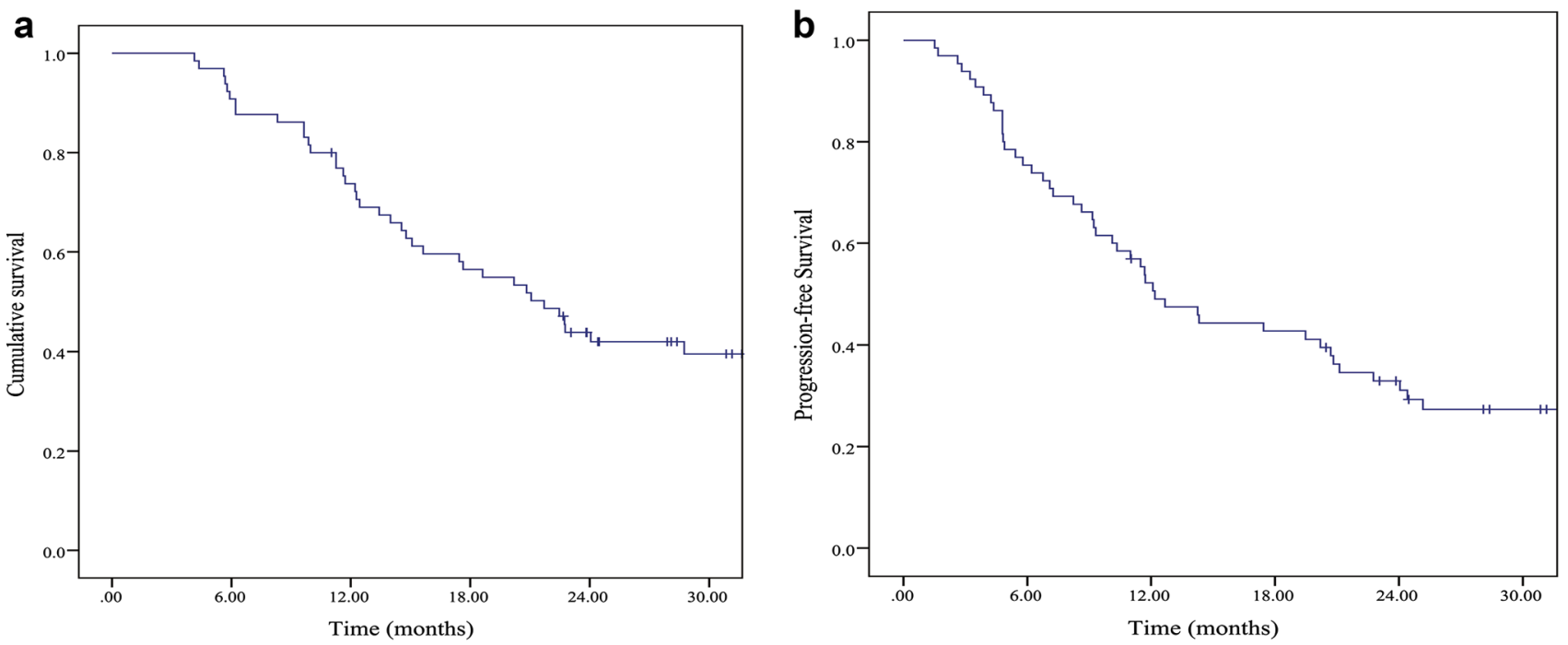

Fig. 3 Overall survival (a) and progression-free survival (b) for 65 patients; the 1- and 2-year overall survival rates were $73.7 \%$ and $42.0 \%$, respectively. 1- and 2-year progression-free survival rates were $50.6 \%$ and $31.1 \%$, respectively

2-year OS was $32.1 \%$ for the newly diagnosed group and $53.3 \%$ for the postoperative recurrence group, with median survival of 18.6 and 28.7 months, respectively $(P=0.155)$. The median OS was 24.0 months for the radiation dose of $61.2 \mathrm{~Gy}$ and 14.7 months for the dose of 50.4 Gy $(P=0.204)$. The 2-year OS rate was $42.8 \%$ for the squamous carcinoma and $50.0 \%$ for adenocarcinoma $(P=0.274)$. A total of 46 patients $(70.7 \%)$ had treatment failure. Thirteen patients had only locoregional failure, 28 patients had only distant metastasis, 4 patients had concurrent locoregional/distant failure, and 1 patient failed therapy as a result of heart disease. The treatment failure patterns of the 46 patients are presented in Table 2. At the time of analysis, 32 patients had distant metastases. The sites of metastases comprised the lungs (23 patients, $35.3 \%$ ), distant lymph nodes (19 patients, 29.2\%), bones (11 patients, $16.9 \%)$, liver (10 patients, $15.3 \%$ ), pleura (4 patients, $6.1 \%$ ), skin (2 patients, 3.0\%), spleen (2 patients, $3.0 \%$ ) and brain (2 patients, $3.0 \%$ ). Among the locoregional failures in 16 patients, 11 patients had local regional recurrence and 5 patients had persistent local/regional disease.

Table 2 Location of disease at first treatment failure

\begin{tabular}{lrr}
\hline & $n$ & $\%$ \\
\hline Local/regional only & 13 & 20.0 \\
Distant only & 28 & 43.0 \\
Local, regional and distant & 4 & 6.1 \\
Other & 1 & 1.5 \\
Total & 46 & 70.7 \\
\hline
\end{tabular}

\section{Toxicity}

The toxicity and tolerability of the therapy were evaluated in all patients. The toxicities of chemoradiotherapy (graded according to NCI-CTC 4.0) are outlined in Table 3. Eighteen patients (27.6\%) experienced grade 3 or higher severe events in this study. The most frequent acute adverse events for grades 3 and 4 were neutropenia, thrombocytopenia and oesophagitis. Grades 3 and 4 neutropenia were observed in $12(18.4 \%)$ and 1 (6.1\%) patients, respectively. Grades 3 and 4 thrombocytopenia, oesophagitis, anaemia, fatigue and pneumonitis occurred in 3/65 (4.6\%), 3/65 (4.6\%), 1/53 (1.5\%), 1/65 (1.5\%) and 1/65 (1.5\%) cases, respectively. The other types of adverse reactions related to treatment were mild and included hypoalbuminaemia, peripheral sensory neuropathy and pericardial effusion. No allergic reaction was recorded in any of the patients. The grade 3 radiation dermatitis rate was $1.5 \%$. There were 3 patients who experienced oesophageal fistula.

\section{Discussion}

In our phase II study, the regimen of weekly paclitaxel and carboplatin with concurrent radiotherapy was a promising approach to treating advanced oesophageal cancer in terms of the OS, local control rate and low rate of side effects. Toxicities of grade 3 or higher were $27.6 \%$ (18/65). The common adverse reactions were bone marrow suppression, oesophagitis and fatigue. The 1- and 2-year OS rates were 73.7 and $42.0 \%$, respectively. The median OS time was 21.7 months. The 1- and 2-year PFS rates were 50.6\% and $31.1 \%$, respectively. 
Table 3 Treatment toxicity

\begin{tabular}{|c|c|c|c|c|}
\hline $\begin{array}{l}\text { Grade } \\
N=65\end{array}$ & $\begin{array}{l}1 \\
n(\%)\end{array}$ & $\begin{array}{l}2 \\
n(\%)\end{array}$ & $\begin{array}{l}3 \\
n(\%)\end{array}$ & $\begin{array}{l}4 \\
n(\%)\end{array}$ \\
\hline Neutropenia & $19(29.2)$ & $20(30.7)$ & $12(18.4)$ & $4(6.1)$ \\
\hline Thrombocytopenia & $19(29.2)$ & $10(15.3)$ & $3(4.6)$ & 0 \\
\hline Anaemia & $27(41.5)$ & $15(23.0)$ & $1(1.5)$ & 0 \\
\hline Vomiting & $7(10.7)$ & $4(6.1)$ & $1(1.5)$ & 0 \\
\hline Fever & $1(1.5)$ & $2(3.0)$ & 0 & 0 \\
\hline Fatigue & $9(13.8)$ & $6(9.2)$ & $1(1.5)$ & 0 \\
\hline Alopecia & $10(15.3)$ & $6(9.2)$ & 0 & 0 \\
\hline Hypoalbuminaemia & $13(20.0)$ & $3(4.6)$ & 0 & 0 \\
\hline $\mathrm{ALT}^{\mathrm{a}}$ & $3(4.6)$ & 0 & 0 & 0 \\
\hline Radiation dermatitis & $8(12.3)$ & $8(12.3)$ & $1(1.5)$ & 0 \\
\hline Oesophagitis & $19(29.2)$ & $19(29.2)$ & $3(4.6)$ & 0 \\
\hline Hypocalcaemia & $8(12.3)$ & $1(1.5)$ & 0 & 0 \\
\hline Hypomagnesaemia & $2(3.0)$ & $1(1.5)$ & 0 & 0 \\
\hline Hyponatraemia & $8(12.3)$ & 0 & 0 & 0 \\
\hline Hypophosphataemia & $4(6.1)$ & 0 & $1(1.5)$ & 0 \\
\hline Constipation & $2(3.0)$ & $6(9.2)$ & 0 & 0 \\
\hline Diarrhoea & $1(1.5)$ & $2(3.0)$ & 0 & 0 \\
\hline $\begin{array}{l}\text { Peripheral sensory neu- } \\
\text { ropathy }\end{array}$ & $2(3.0)$ & $12(18.4)$ & 0 & 0 \\
\hline Pneumonitis & $5(7.6)$ & $7(10.7)$ & $1(1.5)$ & 0 \\
\hline Hypopotassaemia & $3(4.6)$ & 0 & 0 & 0 \\
\hline Hoarseness & $7(10.7)$ & $2(3.0)$ & 0 & 0 \\
\hline Ventricular arrhythmia & $3(4.6)$ & 0 & 0 & 0 \\
\hline Pericardial effusion & $4(6.1)$ & 0 & 0 & 0 \\
\hline
\end{tabular}

Toxicity was graded according to the National Cancer Institute Common Toxicity Criteria, version 4.0

${ }^{a}$ Alanine aminotransferase increased

Compared to cisplatin and 5-fluorouracil (PF) with concurrent radiotherapy in RTOG 85-01 and RTOG 94-05, the regimen of paclitaxel and carboplatin produced less toxicity and increased OS $[3,12,13]$. In the RTOG 85-01 study, the median survival duration was 14.1 months with a 5-year survival rate of $26 \%$ in the chemoradiotherapy treatment group [3, 12]. Additionally, grade 3-4 adverse reactions occurred in $46 \%$ of the patients. In the RTOG94-05 study, patients were treated with radiotherapy doses of 64.8 and 50.4 Gy with PF, and the median survival times were 13.0 and 18.1 months, respectively [13]. The incidences of grade 3-5 acute toxicity in the high-dose and low-dose groups were 76 and $71 \%$, respectively. In our study, the median OS was 21.7 months, and all patients had unresectable or recurrent oesophageal cancer, including 22/34 (64.7\%) previously untreated patients diagnosed in stage IV.

Paclitaxel and cisplatin (TP) regimens are the most commonly reported paclitaxel-based regimens. Compared with cisplatin, the administration of carboplatin is convenient and does not require planned hydration and considerably shortens the duration of outpatient attendance. Adelstein et al. reported the use of TP in a phase II study [14]. The median survival was 15 months for patients with stage T3, N1 or M1 (nodal) oesophageal cancer, lower than that observed in our study. Neutropenia $(95 \%)$ and nausea $(95 \%)$ were the most frequent grade III/IV toxicities, and 16 patients (40\%) experienced neutropenic fever, which must be addressed. This toxicity is significant and was higher than that in our study. Lawrence et al. reported a phase II study of neoadjuvant chemoradiotherapy with weekly dose TP compared with irinotecan plus cisplatin for resectable oesophageal adenocarcinoma [15]. The median OS survival time in the TP group was 21 months; however, acute toxicities grade 3-4 occurred in $65.2 \%$, with relatively higher haematological toxicity. In the RTOG 0113 study, one group was treated with paclitaxel plus cisplatin and a radiotherapy dose of $50.4 \mathrm{~Gy}$, and grades 3 and 4 adverse reactions occurred in 43 and $40 \%$ of the patients, respectively [16]. The TP regimen might have higher haematological toxicity, similar to the results of our previous phase II study of concurrent chemoradiotherapy for patients with inoperative oesophageal cancer [17]. In our study, the grade $\geq 3$ haematological toxicity rate was $27.6 \%$, lower than the results reported in a TP-based group study. The median OS was 21.7 months, which was comparable with the TP regimen studies reported.

The CROSS study showed that neoadjuvant chemoradiation combined with carboplatin and paclitaxel concurrent with radiation significantly prolonged OS, and did not increase surgery-related mortality compared with surgery alone [18]. Thus, preoperative chemoradiotherapy with TC regimen is considered to be a standard of care among patients with resectable oesophageal cancer. Meerten et al. reported a phase 2 study of definitive chemoradiotherapy with weekly dose paclitaxel $\left(50 \mathrm{mg} / \mathrm{m}^{2}\right)$ and carboplatin $($ AUC $=2)$ [19]. The median OS was 17 months. The grade 3 and 4 adverse events were neutropenia (16\%), oesophagitis (12\%), fatigue (8\%) and pneumonitis (2\%). Honing et al. retrospectively compared definitive chemoradiotherapy with TC to PF [20]. The median OS and PFS were 13.8 and 9.7 months, respectively. A higher percentage of patients with TC completed treatment compared with the PF group ( $82 \%$ vs $57 \%, P=0.010$ ). Van Ruler et al. retrospectively reviewed 66 patients treated with chemoradiation therapy with TC regimen [21]. The median OS was 13.1 months. 91 and $82 \%$ of patients, respectively, completed the full course of radiotherapy and 5 courses. In our study, the concurrent chemoradiotherapy completed was $90.7 \%$, and the median OS was 21.7 months. Our results are comparable in terms of completed treatment and survival using the same chemoradiotherapy regimens. 
Few studies have evaluated definitive chemoradiation therapy that included 3 cytotoxic drugs [22, 23]. Miyazaki et al. reported a phase II study of definitive chemoradiation therapy with docetaxel, cisplatin and 5-fluorouracil (DCF) in advanced oesophageal cancer [22]. The 2-year OS rate and median survival time were $52.9 \%$ and 24.7 months, respectively. However, the incidence of leukopenia and neutropenia was 91.9 and $75.7 \%$, respectively. The KDOG 0501-P2 trial reported the use of DCF in a phase II study; the median survival was 29.0 months with a survival rate of $43.9 \%$ at 3 years [23]. Grade 3 or higher major toxicity consisted of leukopenia (71.4\%), neutropenia (57.2\%) and oesophagitis (28.6\%). The clinical complete response (cCR) rate ranged from 54.1 to $52.4 \%$. The DCF regimen appears to be highly active and to have relatively higher toxicity.

The SCOPE1 trial was a phase $2 / 3$ study assessing the benefits of adding cetuximab to definitive chemoradiation therapy (capecitabine + cisplatin + radiation) to treat patients with localised oesophageal cancer [24]. The 2-year survival was $41.3 \%$ in the CRT plus cetuximab group and $56.0 \%$ in the CRT only group. The CRT plus cetuximab group also had shorter median OS (22.1 vs 25.4 months, respectively; $P=0.035$ ) and more nonhaematological grade 3 or 4 toxicities ( $79 \%$ vs $63 \%$, $P=0.004)$. In our study, adverse events such as myelosuppression and oesophagitis were relatively mild.

Toxicity rates for TC in our study were comparable with the rates in the paclitaxel-based trial. The grade $\geq 3$ haematological toxicity rates of TP with concurrent radiotherapy were $47-95 \%[14,15,17,25]$. In our study, $27.6 \%$ of patients experienced grade 3-4 hematological toxicities and $12.3 \%$ non-hematological toxicities, which was lower than that of the TP regimen. In our previous phase II study of paclitaxel and fluorouracil (TF) with concurrent radiotherapy, the occurrence of grade $\geq 3$ or higher severe events was $32 \%$ and that of haematological events was $16.9 \%$ [26]. However, 8 patients experienced fever and 2 patients died from radiation pneumonitis in the TF regimen. Long-term continuously infused 5-FU might lead to higher incidence of oesophagitis and fever.

The limitations of our study are as follows. (1) The homogeneity of the patients in the group was poor; there were patients in phases II-III and IV, and there was postoperative recurrence. (2) The radiation dose was not uniform. Although the standard radiotherapy dose is $50.4 \mathrm{~Gy}$ in Europe and the United States, the radiotherapy dose for oesophageal cancer in China is often $\geq 60 \mathrm{~Gy}$. Only normal tissue could not tolerate the use of 50.4 Gy in our study. Wang et al. reported that patients who received a radiation dose of $\geq 50 \mathrm{~Gy}$ had a better outcome than those who received $<50$ Gy [27]. Additionally, there was no statistical difference in the survival rates of patients with adenocarcinoma and squamous cell carcinoma, probably because the number of patients with adenocarcinoma was too small.

In conclusion, our study indicates that weekly infused paclitaxel and carboplatin, followed by 2-cycle consolidation chemotherapy, is a promising regimen with good tolerability for patients with oesophageal cancer. The limitation of our study is the small sample size. A randomised phase III trial (NCT02459457) with larger sample sizes is currently examining the efficacy of paclitaxel in combination with cisplatin, carboplatin or 5-fluorouracil concurrent with radiotherapy is on-going at our hospital.

Acknowledgements This study was financially supported by the National Natural Science Foundation of China Research, China (Grant No. 21172043 and No. 21441010) and Natural Science Foundation of Minhang District, Shanghai (Grant No. 2014MHZ080).

\section{Compliance with ethical standards}

Conflict of interest The authors declare that they have no conflicts of interest.

Open Access This article is distributed under the terms of the Creative Commons Attribution 4.0 International License (http://creativeco mmons.org/licenses/by/4.0/), which permits unrestricted use, distribution, and reproduction in any medium, provided you give appropriate credit to the original author(s) and the source, provide a link to the Creative Commons license, and indicate if changes were made.

\section{References}

1. Siegel RL, Miller KD, Jemal A (2017) Cancer statistics, 2017. CA Cancer J Clin 67(1):7-30

2. Pennathur A, Gibson MK, Jobe BA et al (2013) Oesophageal carcinoma. Lancet 381:400-412

3. Cooper JS, Guo MD, Herskovic A et al (1999) Chemoradiotherapy of locally advanced esophageal cancer: long-term follow-up of a prospective randomized trial (RTOG 85-01). Radiation Therapy Oncology Group. JAMA 281:1623-1627

4. al-Sarraf M, Martz K, Herskovic A et al (1997) Progress report of combined chemoradiotherapy versus radiotherapy alone in patients with esophageal cancer: an intergroup study. J Clin Oncol 15(1):277-284

5. Ajani JA, Ilson DH, Daugherty K et al (1994) Activity of taxol in patients with squamous cell carcinoma and adenocarcinoma of the esophagus. J Natl Cancer Inst 86:1086-1091

6. Choy H, Rodriguez FF, Koester S et al (1993) Investigation of taxol as a potential radiation sensitizer. Cancer 71:3774-3778

7. Choy H, Akerley W, Safran H et al (1998) Multiinstitutional phase II trial of paclitaxel, carboplatin, and concurrent radiation therapy for locally advanced non-small-cell lung cancer. J Clin Oncol 16:3316-3322

8. Choy H, Devore RF, Hande KR et al (2000) A phase II study of paclitaxel, carboplatin, and hyper fractionated radiation therapy for locally advanced inoperable non-small-cell lung cancer (a Vanderbilt Cancer Center Affiliate Network Study). Int J Radiat Oncol Bio Phys 47:931-937 
9. Lau D, Leigh B, Gandara D et al (2001) Twice-weekly paclitaxel and weekly carboplatin with concurrent thoracic radiation followed by carboplatin/paclitaxel consolidation for stage III nonsmall-cell lung cancer: a California Cancer Consortium phase II trial. J Clin Oncol 19(2):442-447

10. van Meerten E, Muller K, Tilanus HW et al (2006) Neoadjuvant concurrent chemoradiation with weekly paclitaxel and carboplatin for patients with oesophageal cancer: a phase II study. Br J Cancer 94(10):1389-1394

11. van Hagen P, Hulshof MC, van Lanschot JJ et al (2012) Preoperative chemoradiotherapy for esophageal or junctional cancer. $\mathrm{N}$ Engl J Med 366(22):2074-2084

12. Herskovic A, Martz K, al-Sarraf M et al (1992) Combined chemotherapy and radiotherapy compared with radiotherapy alone in patients with cancer of the esophagus. N Engl J Med 326(24): 1593-1598

13. Minsky BD, Pajak TF, Ginsberg RJ et al (2002) INT 0123 (Radiation Therapy Oncology Group 94-05) phase III trial of combinedmodality therapy for esophageal cancer: high-dose versus standard-dose radiation therapy. J Clin Oncol 20:1167-1174

14. Adelstein DJ, Rice TW, Rybicki LA et al (2000) Does paclitaxel improve the chemoradiotherapy of locoregionally advanced esophageal cancer? A nonrandomized comparison with fluorouracil-based therapy. J Clin Oncol 18:2032-2039

15. Kleinberg LR, Catalano PJ, Forastiere AA et al (2016) Eastern Cooperative Oncology Group and American College Of Radiology Imaging Network randomized phase 2 trial of neoadjuvant preoperative paclitaxel/cisplatin/radiation therapy (RT) or irinotecan/cisplatin/RT in esophageal adenocarcinoma: long-term outcome and implications for trial design. Int J Radiat Oncol Biol Phys 94:738-746

16. Ajani JA, Winter K, Komaki R et al (2008) Phase II randomized trial of two nonoperative regimens of induction chemotherapy followed by chemoradiation in patients with localized carcinoma of the esophagus: RTOG 0113. J Clin Oncol 26:4551-4556

17. Tang H, Ma H, An SM et al (2016) A phase II study of concurrent chemoradiotherapy with paclitaxel and cisplatin for inoperable esophageal squamous cell carcinoma. Am J Clin Oncol 39(4):350-354
18. Shapiro J, van Lanschot JJB, Hulshof MCCM et al (2015) Neoadjuvant chemoradiotherapy plus surgery versus surgery alone for oesophageal or junctional cancer (CROSS): long-term results of a randomised controlled trial. Lancet Oncol 16(9):1090-1098

19. Marten EV, van Rij C, Tesselaar ME et al (2010) Definitive concurrent chemoradiation (CRT) with weekly paclitaxel and carboplatin for patients (pts) with irresectabe esophageal cancer: a phase II study. J Clin Oncol 28:e14508

20. Honing J, Smit JK, Muijs CT et al (2014) A comparison of carboplation and paclitaxel with cisplatinum and 5-fluorouracil in definitive chemoradiation in esophageal cancer patients. Ann Oncol 25:638-643

21. van Ruler MA, Peters FP, Slingerland M et al (2017) Clinical outcomes of definitive chemoradiotherapy using carboplatin and paclitaxel in esophageal cancer. Dis Esophagus 30:1-9

22. Miyazaki T, Sohda M, Tanaka N et al (2015) Phase I/II study of docetaxel, cisplatin, and 5-fluorouracil combination chemoradiotherapy in patients with advanced esophageal cancer. Cancer Chemother Pharmacol 75(3):449-455

23. Higuchi K, Komori S, Tanabe S et al (2014) Definitive chemoradiation therapy with docetaxel, cisplatin, and 5-fluorouracil (DCF-R) in advanced esophageal cancer: a phase 2 trial (KDOG 0501-P2). Int J Radiat Oncol Biol Phys 89(4):872-879

24. Crosby T, Hurt CN, Falk S et al (2013) Chemoradiotherapy with or without cetuximab in patients with oesophageal cancer (SCOPE1): a multicentre, phase $2 / 3$ randomised trial. Lancet Oncol 14:627-637

25. Ilson DH, Forastiere A, Arquette M et al (2000) A phase II trial of paclitaxel and cisplatin in patients with advanced carcinoma of the esophagus. Cancer J 6(5):316-323

26. Xia Y, Li YH, Chen Y et al (2017) A phase II study of concurrent chemoradiotherapy combined with a weekly paclitaxel and 5-fluorouracil regimen to treat patients with advanced esophageal carcinoma. Radiat Oncol 12:47

27. Wang S, Liao Z, Chen Y et al (2006) Esophageal cancer located at the neck and upper thorax treated with concurrent chemoradiation: a single-institution experience. J Thorac Oncol 1:252-259 